\title{
Plasma miR-126 levels and its genomic polymorphism SNP rs4636297 in Type 2 diabetes
}

\author{
Monireh Rezai ${ }^{a}$, Shirin Shahbazi ${ }^{a^{*}}$, Nasrin Mansournia ${ }^{b}$ \\ ${ }^{a}$ Department of Medical Genetics, Faculty of Medical Sciences, Tarbiat Modares University, Tebran, Iran \\ ${ }^{b}$ Department of Endocrinology and Metabolism, AJA University of Medical Sciences, Tehran, Iran
}

Received 5th September 2019 / Accepted 1st January 2020

\begin{abstract}
Since the variation of microRNAs expression has been described in type 2 diabetes, we aimed to evaluate the plasma levels of miR-126 with known roles in the angiogenesis. Genomic polymorphism of miR-126 (SNP rs4636297) was also assessed as it is involved in the processing of pri-miR-126 to mature microRNA.Genotyping of the SNP rs4636297 was carried out by PCR-RFLP using HaeII enzyme. Realtime PCR assay was applied to assess miR-126 expression, which the fold change in gene expression was calculated by the Relative Expression Software Tool (REST). Data analysis was performed using exactlike logistic regression, Fisher exact test and the elrm package with the $\mathrm{R}$ software. Our data indicated that the group with BMI greater than 30 was at greater risk of diabetes (odds ratio $=14.4, p$ value $=0.00$ ), compared with the BMI=19-25. The mean fold decrease in miR-126 gene expression in diabetic samples relative to normal samples was 0.653 (95\% confidence interval: 0.012 - 18.765). All patients with the lowest levels of miR-126, had experienced diabetic complications. Considering the AA genotype as the reference, the odds ratio of diabetic complications for the GG and AG genotype were 1.2 and 1.43, respectively. miR-126 is one of the key factors in predisposition to diabetes and diabetic complication. Since the lowest expression of miR-126 leads to the diabetic complications it can be concluded that genetic predisposing factors should be considered in the management and treatment of Type 2 diabetes.
\end{abstract}

Keywords: human plasma, miR-126, rs4636297, Type 2 diabetes, gene expression, RFLP

\section{INTRODUCTION}

Diabetes mellitus consists of heterogeneous metabolic diseases that are characterized by hyperglycemia due to defective insulin secretion, its function, or both (Roder et al., 2016). Type 2 diabetes (T2DM) is the most common type of the disease with an incidence of 90-95\% (Chen et al., 2014). Reflected by several studies, microRNAs variations have been implicated in the progress of T2DM and its complications (Deiuliis et al., 2016; Lee et al., 2016; Simpson et al., 2016).

MicroRNAs are small noncoding RNAs composed of 21-23 nucleotides that act as the key regulators of gene expression and biological processes (He et al., 2017). Previous studies have reported the reduction of miR-126 expression in T2DM patients with or without complications (Kuhnert et al., 2008; Zampetaki et al., 2010). miR126 is expressed by the vascular endothelial cells and plays an important role in angiogenesis and vascular integrity. This microRNA is a positive regulator of endothelial cell response to vascular endothelial growth factor (VEGF); therefore, its reduction results in resistance to VEGF and defective endothelial cells function. miR-126 gene is located at human chromosome 9 on the seventh intron of the EGFL7 (epidermal growth factorlike protein 7) gene (Meister et al., 2010). This microRNA directly targets phosphoinositide-3-

*Author for correspondence: Shirin Shahbazi, Department of Medical Genetics, Faculty of Medical Sciences, Tarbiat Modares University, Tehran, Iran. Email - sh.shahbazi@modares.ac.ir 
kinase regulatory subunit 2 (PI3KR2) and sprouty-related protein-1 (SPRED-1), two negative regulators of the VEGF signaling pathway. Accordingly, it enhances the signaling of phosphoinositide-3-kinase (PI3K) and VEGFdependent MAP kinase and consequently, affects vascular integrity and angiogenesis (Fish et al., 2008; Liu et al., 2009). One of the main targets of the miR-126 is its host gene; EGFL7. EGFL7 protein is secreted from endothelial cells and is involved in migration and adhesion of endothelial and smooth muscle cells (Kuhnert et al., 2008).

miR-126 also inhibits the expression of vascular adhesion molecule 1 (VCAM-1) in endothelial cells and decreases the leukocytes adhesion which counteract tissue inflammation. Recently, miR-126 was suggested as a therapeutic tool to decrease neuroinflammation via VCAM1 reduction (Cerutti et al., 2017).

Given the role of miR-126 in regulating cell migration, vascular adhesion, vascularization and subsequently wound healing, it should be considered as a serious candidate for the treatment of vascular defects, including diabetic complications (Qu et al., 2018).

Genetic variants are involved in MicroRNA expression. Only one single nucleotide polymorphism (SNP) has been identified for mir126. This SNP is a substitution of the twentyfourth base, known as the SNP rs4636297. This SNP prevents the processing of pri-microRNA to mature microRNA, resulting in a reduced suppression of the various targets of miR-126. It has been shown that, despite higher $G$ allele expression compared to A allele, the efficacy of $G$ allele processing was only $16 \%$ of the A allele processing efficacy. This remarkable difference, roots in pri-mir-126-G condensed structure prevents the access of drosha enzyme. As a result, the amount of mature miR-126 in cells containing A allele is higher than cells bearing $G$ allele(Harnprasopwat et al., 2010).

In the present study, we evaluated the frequency of genomic SNP rs4636297 and the miR-126 gene expression in T2DM patientsin the Iranian population for the first time. We have also studied the association between the T2DM complications and miR-126 plasma levels.

\section{MATERIALS AND METHODS}

\section{Participants}

T2DM patients were selected according to the latest criteria of the World Health Organization. Briefly, patients have considered T2DM if: 1) fasting plasma glucose (FPG) was equal to or higher than $126 \mathrm{mg}$ percent, 2) hyperglycemia symptoms present along with plasma glucose in a casual testing, equal to or greater than $200 \mathrm{mg}$ percent, 3) two-hour, 75-gram oral glucose tolerance test (OGT'T) equal to or greater than $200 \mathrm{mg}$ percent. Based on the mentioned criteria 34 T2DM and 38 non-diabetic individuals aged 45-64 years were selected among referrals to Imam Reza Hospital, Tehran, Iran.

In order to exclude the pre-diabetic subjects, the control group was precisely evaluated by a specialist in the field. Individuals were enrolled in the study as the controls who had no symptoms of diabetes in the two previous clinical and laboratory examinations and none of their firstdegree relatives has a medical history of diabetics.

The information on age, sex, weight, height, and the family history of diabetes was collected. Applying a questionnaire, clinical and laboratory characteristics and complications including nephropathy, neuropathy, retinopathy, and cardiovascular disorder were also recorded in case group. BMI of all participants calculated by $\mathrm{kg}$ (weight) $/ \mathrm{m}^{2}$ (height) formula.

Following informed consent obtaining, $2 \mathrm{ml}$ of venous blood samples were collected in EDTA-containing tubes. Blood samples were centrifuged for $5 \mathrm{~min}$ at $1000 \mathrm{xg}$ and plasma was transferred to RNase free tubes. Using spectrophotometer (Biochrom, WPA), oxyhemoglobin adsorption was calculated at 414 $\mathrm{nm}$ to determine the purity of the plasma and then the samples were stored at $-80^{\circ} \mathrm{C}$ until further investigation.

\section{Genotyping by PCR- RFLP}

DNA extraction was done by salting out method on blood cells obtained during centrifuge steps for plasma isolation. Specific primers were designed using the Gene Runner software and verified using BLAST (Basic Local Alignment Search Tool) website. The final volume of the PCR was $20 \mu$ l, containing $10 \mu \mathrm{l}$ of master mix 
(Taq DNA polymerase 2X Master Mix RED, PishgamBiotech, IRAN), $1 \mu$ l of each primers, $8 \mu 1$ of double distilled water and $1 \mu \mathrm{l}$ of DNA. PCR program was then performed in 30 cycles at $95^{\circ} \mathrm{C}$ for 5 minutes, $95^{\circ} \mathrm{C}$ for 30 seconds, $60^{\circ} \mathrm{C}$ for 30 seconds, $72^{\circ} \mathrm{C}$ for 40 seconds, $72^{\circ} \mathrm{C}$ for 10 minutes followed by visualization of DNA by $1.5 \%$ agarose gel. To identify SNP rs4636297 genotypes, RFLP method was applied using HaeII enzyme (Thermo Scientific FastDigest HaeII (BfoI), PishgamBiotech, IRAN). The reaction mixture consisted of $2 \mu \mathrm{l}$ buffer, 0.5 $\mu \mathrm{H}$ HaeII enzyme, $8.5 \mu \mathrm{l}$ double distilled water and $10 \mu \mathrm{l}$ PCR product in a final volume of $20 \mu \mathrm{l}$, which was incubated at $37^{\circ} \mathrm{C}$ for $4-5$ hours.

\section{MicroRNAs extraction and real-time PCR}

Total RNA extraction from plasma was performed using RNX plus (Sinaclon, IRAN) according to the manufacturer's instructions. cDNA was reverse transcribed from $10 \mathrm{ng}$ of total RNA using the miR-126 specific stem-loop primer. miR-16 was selected as the intrinsic reference gene for the normalization of miR-126 expression. Reverse transcription was performed with the BioFact ${ }^{\mathrm{TM}}$ RT Series (Noavaranteb, Iran). MicroRNAs specific forward primer and universal reverse primer were employed for quantitative real-time PCR amplification. Amplification of miR-126 and miR-16 was performed on StepOne Real-Time PCR System using $10 \mu \mathrm{l}$ of the Biofact Syber-green high-Rox master mix (Noavaranteb, Iran), $1 \mu \mathrm{L}$ of forward primer, $1 \mu \mathrm{l}$ of reverse primer, $6 \mu \mathrm{l}$ of double distilled water and $2 \mu \mathrm{l}$ of cDNA in the final volume of $20 \mu \mathrm{l}$. The program was defined as $95^{\circ} \mathrm{C}$ for 15 minutes, $95^{\circ} \mathrm{C}$ for 20 seconds, and $60^{\circ} \mathrm{C}$ for 40 seconds. No Template Control (NTC) reactions were also included in the experiments. All qRT-PCR reactions were performed in duplicate.

\section{Statistical analysis}

Fold change in gene expression was determined using the Relative Expression Software Tool (REST $\left.{ }^{\circledR}\right)$. To investigate the association of SNP rs4636297 genomic polymorphism with the risk of T2DM and T2DM complications due to the low sample size and subgroups, exact-like logistic regression and fisher exact test were applied by $\mathrm{R}$ software, using the elrm package. The regression model was adjusted based on age and gender variables. As smoking and blood pressure influence T2DM, the analyses were adjusted for these confounding factors in the exact logistic model. However, the results remained unchanged, suggesting that our analysis is not markedly affected by these risk factors.

\section{RESULTS}

\section{Patient's data}

In order to cluster the data in case and control groups, the groups were matched in terms of gender and age. The mean age in the cases and controls were $58 \pm$ and $57 \pm 6.086$, respectively. The study of the link between BMI and the risk of T2DM revealed that the group with BMI greater than 30 was at greater risk of T2DM (OR = 14.4; CI: 2.34-446) compared to the reference group (BMI 19-25) which was statistically significant $(p$ value, 0.00$)$. There were significant differences between cases and controls in terms of fasting glucose and hemoglobin A1c rates, however triglycerides and cholesterol measurements showed no important differences between groups (Table1).

\section{Genotyping of the SNP rs 4636297}

The HaeII enzyme has RG $\downarrow$ CGCY restriction site and $G$ allele substitution with $A$ allele removes the restriction site. As a result, AA genotype had only one fragment of $335 \mathrm{bp}$ and those of the genotype AG displayed three fragments at $225 \mathrm{bp}, 110 \mathrm{bp}$ and 335 bp. GG genotype produced two distinct bands of $225 \mathrm{bp}$ and $110 \mathrm{bp}$. In order to confirm the accuracy of RFLP results, randomly selected samples with $A A, A G$ and $G G$ genotypes were sequenced (Figure 1). Using the information obtained from RFLP, the frequency of A allele and $G$ allele were calculated 0.43 and 0.57 respectively. The frequency of $A$ allele and $G$ allele in healthy subjects were 0.45 and 0.55 , respectively. Regarding the link between genotypes and the general risk of diabetes, the odds ratio for GG genotype was 1.13 (CI: 0.2564.53) and the odds ratio for $A G$ genotype was 1.26 (CI: 0.311-5.91) compared with the AA reference genotype. The risk of diabetic complications for the GG and AG genotype showed a slight 
increase, considering the AA genotype as the reference group $(\mathrm{OR}=1.12, \mathrm{CI}: 0.117-11$ and $\mathrm{OR}=2.13$, CI: 0.22-27.1, respectively). However, the differences were not statistically significant for both genotypes ( $p$-value $>0.05$ ).

Table 1. Clinical characteristics of the study subjects.

\begin{tabular}{lccc}
\hline & $\begin{array}{c}\text { Normal controls } \\
\text { Mean } \pm \text { SD }\end{array}$ & $\begin{array}{c}\text { T2DM } \\
\text { Mean } \pm \text { SD }\end{array}$ & P value \\
\hline Number of subjects & 38 & 24 & - \\
Age (year) & $57 \pm 6.086$ & $58 \pm 5$ & 0.521 \\
BMI (kg/m $\mathrm{m}^{2}$ & $26 \pm 3$ & $29 \pm 3$ & 0.001 \\
Duration of diabetes (month) & - & $76 \pm 96$ & - \\
Familial history (complicated) & - & $44.11 \%$ & - \\
\hline Biochemical tests & & & 0.267 \\
TG* (mmol/L) & $121 \pm 50$ & $183 \pm 253$ & 0.000 \\
FG** (mmol/L) & $91 \pm 6$ & $144 \pm 48$ & 0.408 \\
Cholestrol (mmol/L) & $188 \pm 45$ & $178 \pm 32$ & 0.000 \\
HbA1c*** (\%) & $5 \pm 0$ & $7 \pm 1$ & - \\
\hline Complications & - & & - \\
Complicated & - & $47.1 \%$ & - \\
Nephropathy & - & $0 \%$ & - \\
Neuropathy & - & $8.8 \%$ & - \\
Retinopathy & - & $11.8 \%$ & - \\
Neuropathy + Retinopathy & - & $5.9 \%$ & \\
Cardiovascular & & $20.6 \%$ & \\
\hline
\end{tabular}

*Triglycerides, **Fasting Glucose, ***Hemoglobin A1c



Figure 1. Electropherograms obtained from the three possible genotypes. Above AG, middle AA, and below GG genotypes.

\section{miR-126 gene expression}

Melting curve analysis showed a single specific peak for each microRNAs. Raw amplification data were transferred to LinReg software and used to generate linear standard curves (Figure 2). The mean fold decrease of miR-126 gene expression in T2DM samples relative to normal samples was 0.653 (95\% confidence interval: 0.012 - 18.765; standard error: 0.093 - 4.257). The overall profile of the gene expression variations remained unchanged when the data were adjusted to age and gender. Delta $\mathrm{Ct}(\Delta \mathrm{CT})$ value for each sample was calculated using the formula (Ct target gene $\mathrm{Ct}$ reference gene). Then miR-126 expression was categorized into 3 groups; $\Delta \mathrm{CT}<-3$ with the highest expression, $\Delta \mathrm{CT}$ between -3 to +3 and $\Delta \mathrm{CT}>+3$ with the lowest expression. As indicated in Figure 3, the frequency of $A G$ genotype in the group with the lowest expression of miR-126 $(\Delta \mathrm{CT}>+3)$ was higher than AA genotype; while, there was no GG genotype in this group. Furthermore, the results showed that $44 \%$ of uncomplicated T2DM patients had miR126 expression in the $\Delta C T$ range of $<-3$ (highest 
expression), whereas only $19 \%$ of subjects with T2DM complications expressed miR -126 in $\Delta \mathrm{CT}<-3$ (Figure 4). On the other hand, all of the patients in $\Delta \mathrm{CT}>+3$ group with the lowest expression of miR-126 had experienced a kind of T2DM complications.

Figure 2. Calculation of the real-time PCR efficiencies. Raw amplification data were transferred to LinReg software and used to generate linear standard curves. The PCR efficiencies were calculated for miR-16 andmiR-126 gene expression reactions.

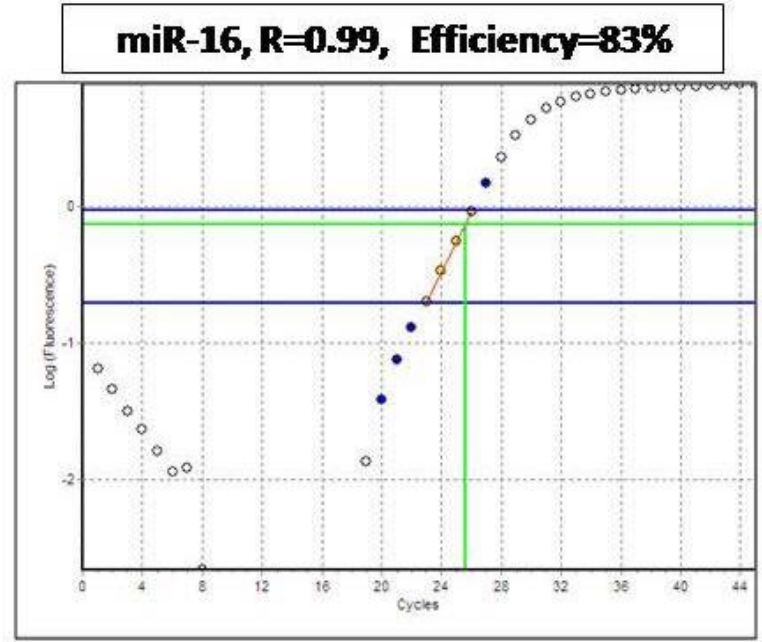

Figure 3. Correlations between genotypes and the expression of miR-126.

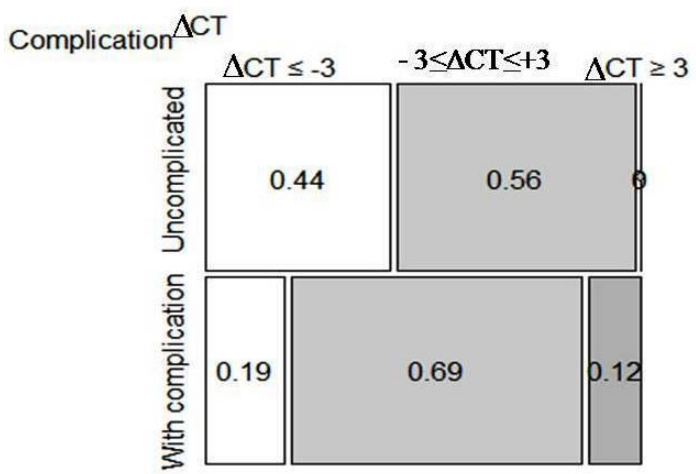

Figure 4. Correlations between the diabetic complications and the expression of miR-126

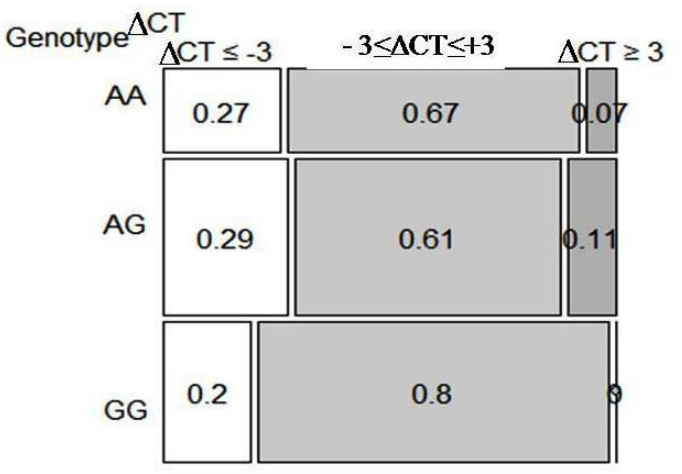

$\operatorname{miR}-126, R=0.99$, Efficiency $=86 \%$

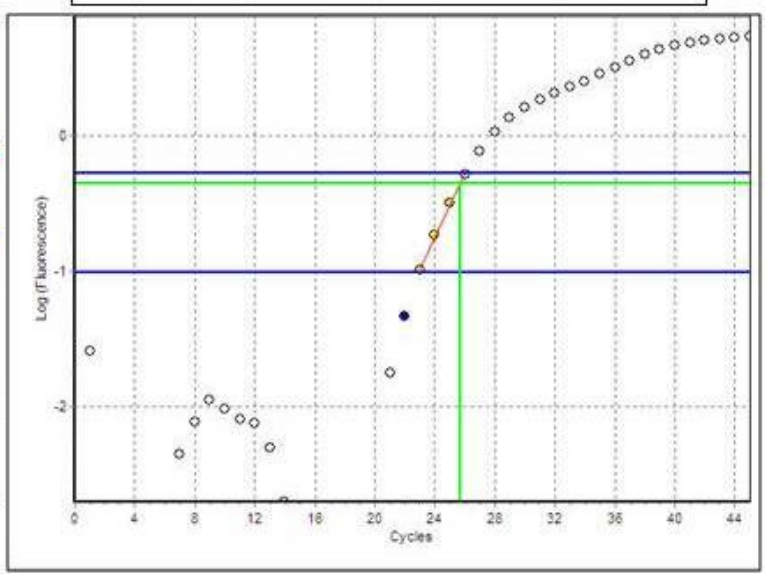

DISCUSSION

The overall pattern of T2DM worldwide indicates that developing countries are at higher risk of the disease. The Tehran Lipid and Glucose Study, which is the most significant national study over the past 15 years in the field of diabetes, indicate that the incidence of T2DM is rapidly increasing due to the lifestyle (Hadaegh et al., 2007). The results of our study also revealed that BMI is significantly associated with an increased risk of T2DM. According to a previous study in Iran, $\mathrm{BMI}$ is an important factor for progression from healthy to pre-diabetes status especially in men (Khaloo et al., 2018). Interestingly, a new study has shown that increased BMI is associated with lower miR-126. This finding is also true in diabetic patients and it can be expected that with increasing weight, the level of miR-126, which has anti-inflammatory function, will be drastically reduced and pave the way for vascular inflammation(Hijmans et al., 2018).

To provide elements to explain inter population variation we focused on miR-126 plasma levels and its genomic SNP rs4636297. Based on our results the A and $G$ allele frequency were 0.43 and 0.57 in T2DM group. A previous study that has been conducted on the association of miR-126 genomic SNP rs4636297 with diabetic 
retinopathy, has reported the $A$ and $G$ allele frequency, 0.40 and 0.60 respectively (McAuley et al., 2015). In different populations the frequency of A allele varies from 0.1 to 0.4 based on the information provided at ENSEMBELE browser, with the maximum A allele frequency of 0.45 . Taken together these results indicate that the Iranian population can be among the populations with the highest frequency of $\mathrm{A}$ allele. Of course, given the sample size, we can't conclude on the allele frequency in Iranian population for sure and this result should be considered as a preliminary. McAuleyet al. also reported a significant relationship between the presence of $A$ allele and STDR (sight-threatening diabetic retinopathy) (McAuley et al., 2015).

We observed an increased odds ratio for GG and AG genotypes compared with AA genotype for the risk of T2DM and T2DM complications, interestingly with a greater susceptibility for AG genotype. To provide elements to explain this observation we analyzed the miR-126 expression in correlation with genotypes. Our finding indicates that among patients with the lowest plasma levels of miR-126, the frequency of $A G$ genotype was higher than AA and GG genotypes. As mentioned before, it has been shown that despite higher expression of the $G$ allele compare to $A$ allele, the processing efficacy of $G$ allele for mature miR-126 synthesis is much lower than cells that express A allele. It can be assumed that, in heterozygote AG genotype, A allele causes lower primary expression and $G$ allele causes reduced processing efficiency of pri-microRNA that simultaneously leads to lower expression of mature miR-126. However, the genotype of SNP rs4636297 was not related to mature miR-126 plasma levels as the majority of AG genotype showed normal expression levels. Functional cell study of the heterozygous AG genotype is needed to reveal whether miR-126 processing efficiency is more profoundly affected than homozygous genotypes.

Several studies have been conducted concerning the expression levels of miR-126 in T2DM compared to healthy subjects (Meng et al., 2012; Olivieri et al., 2015; Zhang et al., 2015). In the present study, the results from real-time PCR showed 0.653 mean fold decrease in miR-126 gene expression which confirmed previous studies. According to the results, we observed that all patients in $\Delta \mathrm{CT}>+3$ group which represents the lowest expression of miR-126 had experienced diabetic complications.

Since miR-126 is a positive regulator of angiogenesis in normal endothelial cells, it could be considered as a key factor in predisposition to T2DM complications. miR-126 promotes VEGF activity and vessel formation by down-regulation of SPRED-1 which is an intracellular inhibitor of angiogenic signals (Wang et al., 2008). Furthermore, one of the main targets of the miR126 is EGFL7 which plays a pivotal role in smooth muscle contraction and endothelial cells migration (Kuhnert et al., 2008). Inactivation of EGFL7/miR-126 robustly impairs developmental angiogenesis and vascular integrity via PI3K and VEGF-dependent MAP kinase pathway (Fish et al., 2008). In addition, PI3K regulation occurs by miR-126 mediated activation of angiopoietin-1 signaling which results in vessel stabilization and maturation (Sessa et al., 2012).

\section{CONCLUSION}

By enhancing VEGF signaling, miR-126 is one of the key factors in predisposition to T2DM and its complications. Reduction in the expression of miR-126 interferes with cell homeostasis, vascular coherence, and wound healing, all of which are related to T2DM complications. Further understanding of the genetic factors such as noncoding RNAs involved in T2DMwill provide preventive strategies as well as effective therapeutic methods leading to appropriate management of the disease. It can be concluded that with considerable healthcare costs and resources allocated to T2DM, in addition to nutrition and lifestyle, other contributors such as genetic factors should be considered in the management of the disease.

\section{ACKNOWLEDGMENTS}

The authors would like to thank Dr. Reza Mahdian Chairman of Molecular Medicine 
department of Pasteur institute of Iran for his support and assistance with this project.

\section{REFERENCES}

Cerutti C., Edwards L. J., de Vries H. E., Sharrack B., Male D. K., \& Romero I. A. 2017. MiR-126 and miR-126* regulate shear-resistant firm leukocyte adhesion to human brain endothelium. Scientific Reports 7: 45284.

Chen H., Li L., Wang S., Lei Y., Ge Q., Lv N., Zhou X., \& Chen C. 2014. Reduced miR-126 expression facilitates angiogenesis of gastric cancer through its regulation on VEGF-A. Oncotarget 5(23): 11873-11885.

Deiuliis J. A. 2016. MicroRNAs as regulators of metabolic disease: pathophysiologic significance and emerging role as biomarkers and therapeutics. International Journal of Obesity 40(1): 88-101.

Fish J. E., Santoro M. M., Morton S. U., Yu S., Yeh R. F., Wythe J. D., Ivey K. N., Bruneau B. G., Stainier D. Y., \& Srivastava D. 2008. miR-126 regulates angiogenic signaling and vascular integrity. Developmental Cell 15(2): 272-284.

Hadaegh F., Zabetian A., Harati H., \& Azizi F. 2007. The prospective association of general and central obesity variables with incident type 2 diabetes in adults, Tehran lipid and glucose study. Diabetes Research and Clinical Practice 76(3): 449-454.

Harnprasopwat R., Ha D., Toyoshima T., Lodish H., Tojo A., \& Kotani A. 2010. Alteration of processing induced by a single nucleotide polymorphism in pri-miR-126. Biochemical and Biophysical Research Communications 399(2): 117-122.

He Y., Ding Y., Liang B., Lin J., Kim T. K., Yu H., Hang H., \& Wang K. 2017. A systematic study of dysregulated microRNA in type 2 diabetes mellitus. International Journal of Molecular Sciences 18(3).

Hijmans J. G., Diehl K. J., Bammert T. D., Kavlich P. J., Lincenberg G. M., Greiner J. J., Stauffer B. L., \& DeSouza C. A. 2018. Influence of overweight and obesity on circulating inflammation-related microRNA. MicroRNA 7(2): 148-154

Khaloo P., Hasheminia M., Tohidi M., Abdi H., Mansournia M. A., Azizi F., \& Hadaegh F. 2018. Impact of 3-year changes in lipid parameters and their ratios on incident type 2 diabetes: Tehran lipid and glucose study. Nutrition \& Metabolism 15: 50.

Kuhnert F., Mancuso M. R., Hampton J., Stankunas K., Asano T., Chen C. Z., \& Kuo C. J. 2008. Attribution of vascular phenotypes of the murine Egfl7 locus to the microRNA miR-126. Development 135(24): 3989-3993.

Lee M. J., Park D. H., \& Kang J. H. 2016. Exosomes as the source of biomarkers of metabolic diseases. Annals of Pediatric Endocrinology \& Metabolism 21(3): 119-125.

Liu B., Peng X. C., Zheng X. L., Wang J., \& Qin Y. W. 2009. MiR126 restoration down-regulate VEGF and inhibit the growth of lung cancer cell lines in vitro and in vivo. Lung Cancer 66(2): 169-175.

McAuley A. K., Dirani M., Wang J. J., Connell P. P., Lamoureux E. L., \& Hewitt A. W. 2015. A genetic variant regulating miR-126 is associated with sight threatening diabetic retinopathy. Diabetes \& Vascular Disease Research 12(2): 133138.

Meister J. \& Schmidt M. H. H. 2010. miR-126 and miR-126*: new players in cancer. The Scientific World Journal 10: 2090-2100.
Meng S., Cao J. T., Zhang B., Zhou Q., Shen C. X., \& Wang C. Q. 2012. Downregulation of microRNA-126 in endothelial progenitor cells from diabetes patients, impairs their functional properties, via target gene Spred-1. Journal of Molecular and Cellular Cardiology 53(1): 64-72.

Olivieri F., Spazzafumo L., Bonafe M., Recchioni R., Prattichizzo F., Marcheselli F., Micolucci L., Mensa E., Giuliani A., Santini G., Gobbi M., Lazzarini R., Boemi M., Testa R., Antonicelli R., Procopio A. D., \& Bonfigli A. R. 2015. MiR21-5p and miR-126a-3p levels in plasma and circulating angiogenic cells: relationship with type 2 diabetes complications. Oncotarget 6(34): 35372-35382.

Qu M.-J., Pan J.-J., Shi X.-J., Zhang Z.-J., Tang Y.-H., \& Yang G.Y. 2018. MicroRNA-126 is a prospective target for vascular disease. Neuroimmunology and Neuroinflammation 5(4).

Roder P. V., Wu B., Liu Y., \& Han W. 2016. Pancreatic regulation of glucose homeostasis. Experimental \& Molecular Medicine 48: $\mathrm{e} 219$.

Sessa R., Seano G., di Blasio L., Gagliardi P. A., Isella C., Medico E., Cotelli F., Bussolino F., \& Primo L. 2012. The miR-126 regulates angiopoietin-1 signaling and vessel maturation by targeting p85beta. Biocbimica et Biophysica Acta 1823(10): 1925-1935.

Simpson K., Wonnacott A., Fraser D. J., \& Bowen T. 2016. MicroRNAs in diabetic nephropathy: from biomarkers to therapy. Current Diabetes Reports 16(3): 35.

Wang S., Aurora A. B., Johnson B. A., Qi X., McAnally J., Hill J. A., Richardson J. A., Bassel-Duby R., \& Olson E. N. 2008. The endothelial-specific microRNA miR-126 governs vascular integrity and angiogenesis. Developmental Cell 15(2): 261-271.

Zampetaki A., Kiechl S., Drozdov I., Willeit P., Mayr U., Prokopi M., Mayr A., Weger S., Oberhollenzer F., Bonora E., Shah A., Willeit J., \& Mayr M. 2010. Plasma microRNA profiling reveals loss of endothelial miR-126 and other microRNAs in type 2 diabetes. Circulation Research 107(6): 810-817.

Zhang T., Li L., Shang Q., Lv C., Wang C., \& Su B. 2015. Circulating miR-126 is a potential biomarker to predict the onset of type 2 diabetes mellitus in susceptible individuals. Biochemical and Biophysical Research Communications 463(1-2): 60-63. 